\title{
Conceptual Design of a Small- and Wide-angle Neutron Scattering Diffractometer/Spectrometer for the Second Target Station of the SNS: Centaur

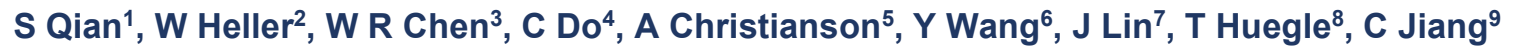 ${ }^{1}$ ORNL ${ }^{2}$ ORNL, ${ }^{3}$ ORNL, ${ }^{4}$ ORNL, ${ }^{5}$ ORNL, ${ }^{6}$ ORNL, ${ }^{7}$ ORNL, ${ }^{8}$ ORNL, ${ }^{9}$ ORNL qianshuo@gmail.com
}

Centaur is an instrument specifically designed and optimized for construction at the Second Target Station (STS) of the SNS at Oak Ridge National Laboratory. As a simultaneous small- and wide-angle neutron scattering (SANS and WANS) instrument with a large dynamic range in reciprocal space, it is optimized to cover length scales from interatomic distances (diffraction) to hundreds of nanometers (small-angle scattering). The design simultaneously provides a high flux and a broad wavelength band as a result of the time-of-flight neutron source at the STS. Further, it can be used as a direct geometry spectrometer for probing the dynamics of relatively large length-scale structures. Centaur will fill a capability gap among the current instruments at ORNL by enabling in situ and operando experiments for kinetic and/or out-of-equilibrium studies of phenomena with time-resolution in seconds in soft matter, polymer science, geology, biology, quantum condensed matter, and other materials sciences. Notably, the WANS and diffraction capability will be unique among SANS instruments in the United States. The spectrometer mode extends the momentum transfer and energy transfer to a lower $\mathrm{Q}$ than typically covered by other spectrometers and provides a unique capability for inelastic SANS studies. Additionally, beam polarization will enable detailed structural and dynamical investigations of magnetic materials and quantum materials. The instrument will play a key role in mastering hierarchical architectures and beyond-equilibrium matter; understanding heterogeneity, interfaces and disorder beyond ideal materials and systems; and advancing imaging capabilities across multiple scales. 